Aus dem path.-anat. Institut in Wien.

\title{
Zur Histologie der Nebenniere des Menschen.
}

Von

Dr. J. P 1 e c n i k.

Hierzu 3 Abbildungen im Text.

Fast alle Autoren, die über den Bau der Nebennieren, sei es beim Menschen, sei es beim Tiere, berichten, erwähnen die eigentümlichen Körner in der Nebennierenrinde, die einige als Fett, andere als etwas vom Fett verschiedenes auffassen. Ueber das zeitliche Auftreten derselben dagegen sind nur spärliche Berichte vorhanden.

Die präzisesten Angaben macht diesbezüglich v. Brunn (Ein Beitrag zur Kenntnis des fein. B. d. Nebenniere, Arch. f. mikr. Anat. 1872), indem er schreibt: "dass die Annahme einer Differenzierung in Rinde und Mark durch Ansammlung von Fettkörnchen in den peripherisch gelegenen Zellen dadurch an Boden verliert, dass man in den Nebennieren ălterer Embryonen, wo die Substanzen scharf von einander getrennt sind, wie im erwachsenen Tiere, noch keine mit Körnchen erfüllte Zellen findet.

E. O. Hultgreen u. O. A. Andersson (Studien zur Phys. u. Anat. d. Nebenn., Leipzig., Veit u. Comp., 1899) fanden bei $4,5 \mathrm{~cm}$ langen Kaninchenembryonen in den Nebennierenrindenzellen Körner; diese seien aber bedeutend spärlicher als beim neugeborenen Tiere.

Ich untersuchte Nebennieren menschlicher Embryonen auf den Gehalt an Rindenkörnern; über die Befunde will ich in Kürze berichten. Bei diesen Untersuchungen ergaben sich noch anderweitige Befunde, welche ich anschliessend mitteilen will.

Fixiert wurden die Organe in Müller's Flüssigkeit, in Altmann's Gemisch, Sublimat. Eingebettet wurden sie durch Petrolather in Paraffin. Petroläther eignet sich, nebst Chloroform, zur Einbettung der in Altmann's Gemisch fixierten und osmierten Stückchen am besten, da ich durch später zu berichtende Versuche fand, dass 
Petroläther (oder Chloroform) das schwächste Extractionsmittel für die osmierten Rindenkörner bildet. Zum geringen Teile wurden die Organe auch in Celloidin eingebettet und geschnitten.

Als jüngstes Stadium stand mir ein $5 \mathrm{~cm}$ langer menschlicher Embryo zur Verfügung. Ich fand da die Rindenkörner ziemlich gleichmässig reichlich auf alle Rindenzellen verteilt. Ihre Menge ist geringer als beim Erwachsenen, sie sind auch kleiner als man sie im Durchschnitt beim Erwachsenen findet und variieren untereinander an Grösse ganz unbedeutend; sie haben einen scharfen Contour, eine runde Form und machen im Ganzen (an nach Altmann fixierten Paraffinpräparaten) den Eindruck solider, tiefschwarzer Kügelchen. Sie liegen stets in der äussersten Plasmazone der Zellen; in den Gefässen und Endothelien fand ich in diesem Stadium keine Rindenkörner.

Obwohl man schon bei $5-7 \mathrm{~cm}$ langen Embryonen eine Differenzierung in der Nebennierenrinde sieht, insofern die periphersten Schichten derselben kleinere Zellen gegenüber den mehr zentralen besitzen und sich auch die Kerne derselben stärker tingieren, so tritt doch eine endgültige Differenzierung erst mit 10 und $12 \mathrm{~cm}$ auf. Einerseits findet man bei $12 \mathrm{~cm}$ langen Embryonen schon einzelne chromaffine Markzellen, anderseits ist jene Verdichtung der periphersten Rindenschichte, welche der Stelle der späteren Glomerulosa entspricht, ausgeprägter und die mehr oder weniger reihenweise Anordnung der Zellen in dieser Schichte bereits auffallend.

Markzellenhaufen (Wiesel's Markballen*) findet man in diesem Stadium noch in allen Höhen der Rinde; es scheint die letzte Formierung des Markes erst nach dem drittenEmbryonalmonate stattzufinden. Die grosse Aehnlichkeit zwischen einwandernden Markzellen und den peripheren Rindenzellen ist auffallend; ihre Plasmaarmut und tiefere Kerntinction machen sie im Müllerpräparat untereinander täuschend ähnlich. In nach Altmann fixierten Präparaten findet sich jedoch der völlige Mangel an osmierten Körnchen in den Markzellen im Gegensatz zu dem nicht geringen Körnerreichtum in den Rindenzellen. Dieser

*) Anm. Wiesel: Ueber d. Entw. d. Nebenniere d. Schweines. Anatom. Hefte, H. 50 . 
Körnerreichtum ist im Stadium von $12-15 \mathrm{~cm}$ Länge viel grösser geworden als er vorher war, und es tritt zugleich im allgemeinen eine Verschiebung desselben ein und zwar so, dass die peripheren Rindenzellen an Körnern reicher werden; die Lage der Körner in der Zelle bleibt aber ungeändert, indem sie stets an der aussersten Plasmaperipherie liegen. Sie sind grösser als in den früheren Stadien, und zwar liegen die grössten in den peripheren Schichten. Sie sind stets ganz rund, scharf begrenzt und solid; Lücken oder ăhnliche Bildungen in ihnen kommen nicht vor. Stossen zwei oder mehrere dieser rindenkörnerführenden Zellen zusammen (Parenchymkörper im Sinne älterer Autoren) so findet man im allgemeinen, dass die Berührungsflächen der Zellen keine Rindenkörner zeigen, sondern dass diese nur an den, den Gefässen zugewendeten Teile der Zellen vorkommen; die Kerne liegen năher den Berührungsflächen.

Am reinsten ist der eben beschriebene Typus an den Nebennierenrinden erwachsener Kaninchen zu treffen.

Die mehr central gelegenen Rindenzellen (als Centrum die grossen Gefăsse mit den sie umlagernden Markzellen aufgefasst) sind, wie erwăhnt, grösser als die peripheren, unregelmässiger angeordnet und körnerärmer; die Körner sind auch kleiner. Um so auffallender ist deswegen das Vorkommen von besonders grossen in Querund Flachschnitten runder, sternförmiger oder plump-spindelig erscheinender Zellen in der centralen Rindenschichte, die auffallend viele, grosse, immer ganz runde, osmierte Körner führen. Diese Zellen liegen entweder unmittelbar an den centralen Gefässen, falls um diese keine Markzellen vorkommen, oder bilden die Grenze zwischen Mark und Rinde; diese Grenze ist aber nicht eine von den besprochenen Zellen gebildete $\mathrm{zusammen-}$ $\mathrm{h} a \mathrm{ng}$ e $\mathrm{nde}$ Linie, da die betreffenden Zellen einzeln liegen und nicht untereinander zusammenhängen. Manchmal findet man ausser diesen Zell e $n$ am entsprechenden Ort grobe, runde, osmierte Klumpen liegen, offenbar durch Zusammenfluss der erwăhnten groben Körner entstanden. In entsprechenden, nicht osmierten Präparaten sieht man die Negative dieser Bilder; grosse einfache, oder mehr weniger fein septierte Räume, wobei die Septa im Centrum zusammenfliessen, in welchem man, wenn auch nicht immer, ein rundes, scharf begrenztes Gebilde sieht, das eine verwischte Kernfärbung zeigt und zweifellos der 
veränderte Kern ist. Im späteren embryonalen Stadium (15 cm und mehr) liegen die Verhältnisse ganz analog, der Körnerreichtum in der Rinde hat zugenommen, ebenso die Zabl der centralen, grobe Körner führenden, grossen Zellen, die von den centralen Gefässen entsprechend der Mächtigkeit des Marklagers abgerückt sind.

Fasst man bei Embryonen dieses Alters die mittleren Partien der Rinde ins Auge, so findet man als einen ganz auffallenden Befund - bei mit Altmanns Gemisch fixierten mit Haemalaun oder Vesuvin nachgefärbten Präparaten - in dieser Schichte Zellen, die durch ihr helles Aussehen gegen die Nachbarschaft abstechen. Sie sind (vergleiche Fig. 1 ein nach Altmann fixiertes und mit Vesuvin nachgefärbtes Präparat einer Nebennierenrinde eines $20 \mathrm{~cm}$ langen Embryos) von der Grösse der Nachbarzellen, eher etwas grösser als kleiner - und haben ein lockeres, körniges Plasma und einen sich schwach färbenden

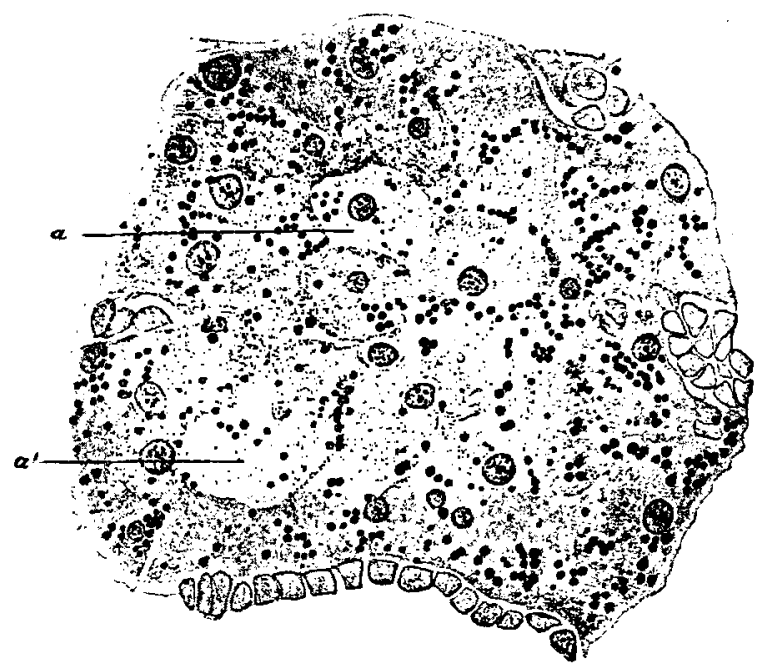

Figur 1.

Kern, manchmal von der Grösse der Zellkerne der Umgebung, im überwiegendem Maasse aber ist der Kern klein, wie atrophisch, oder fehlt ganz. (In Fig. $1 \mathrm{a}^{\mathbf{1}}$.). Färbt man entsprechende Präparate nach Altmann mit Fuchsin, so findet man die anderen Kerne hom ogen und gelblich; diese Kerne aber enthalten radiăr angeordnete Structuren. Die osmierten Körner liegen in diesen 
Zellen ganz unregelmässig im Plasma, nicht an der Peripherie, sie sind auffallend klein und zumeist nicht so tiefschwarz wie die der übrigen Zellen. Im Ganzen haben diese Zellen eine rundliche Form. Ganz analoge Zellen findet man vereinzelt stets, oft sogar in grösserer Häufigkeit in der analogen Schichte der Nebennierenrinde erwachsener Kaninchen. Findet man bei menschlichen Embryonen dieses Alters (bis zum ausgetragenen Neugeborenen) diese Zellen ohne Kerne, aber mit osmierten Körnern, so kann man an ihrer zelligen Natur nicht zweifeln; findet man sie jedoch ohne Kerne und ohne osmierten Inhalt, bloss aus lockerem Plasma in Bruchstücken bestehend, so ist man kaum imstande, sie von den in der Nähe vorhandenen, endothellosen Lücken oder Räumen zu unterscheiden, die keine Blutkörperchen enthalten: solcher Lücken gibt es nun viele, Lücken, deren Wand scheinbar nur von Rindenzellen gebildet wird. $\mathrm{Ob}$ und in welchem Zusammenhang diese Lücken und jene hellen Zellen stehen, ist nicht auffindbar.

Diese Verhăltnisse gelten für die gesamte, spätere embryonale Periode: die inneren Rindenschichten bleiben stets körnerïrmer, als die aussseren und letztere führen auch viel grössere Körner als jene; die Mark-Rindengrenze wird stets durch die erwähnten, grossen, körnerhaltigen Zellen markiert. Ein Unterschied in der Körnermenge der Nebenniere eines todgeborenen Neugeboren und eines einige Tage alten Kindes ist nicht wahrzunehmen. Ebensowenig andern sich die Verhältnisse in der Zeit der postembryonalen Entwicklung wesentlich. Nur bezüglich der Menge der Rindenkörner ist zu bemerken, dass das relative Maximum der letzteren in der zweiten Hälfte des ersten Lebensjahres erreicht wird, welches aber von nun an allerdings unerklarlichen Schwankungen unterliegt.

Nach Henle (Zeitschrift für rationelle Medizin 1865 und Handbuch der Eingeweidelehre) kommt eine Zunahme des Rindenkörnergehaltes beim Menschen mit zunehmendem Alter vor; dasselbe erwähnt A. Ecker für Thiere (Ueber den feineren Bau der Nebennieren 1846, Braunschweig, citiert nach Hultgreen und Andersson). Ich fand stets zu grosse Schwankungen der Rindenkörnermenge bei gleichaltrigen Individuen, um dieser Anschauung beipflichten zu können. Sicher ist es, dass man oft auch im höchsten Alter sehr körnerreiche Nebennierenrinden findet. 
Nach R. May (Virchow's Archiv, Bd. 108, Beitrăge zur pathol. Anatomie der Nebennieren) würden sich Schwankungen aus den Krankheitsprozessen der Individuen erklären lassen, insofern eine pareuchymatöse Degeneration der Nebennieren bei acuten Infectionsprozessen und eine fettige bei allgemeinen Stauungsprozessen vorkommt. May scheint dabei das Alter der untersuchten Individuen nicht näher berücksichtigt zu haben; auch gibt er seine Untersuchungstechnik nicht an. Nach meinen Befunden dürfte die May'sche Anschauung kaum wahrscheinlich sein.

Im Prinzip möchte ich darauf aufmerksam machen, dass ich in Nebennieren, die sehr körnerreich waren, und von denen nach Formalinfixierung mit dem Gefriermikrotom gewonnene Schnitte auf $24^{\mathrm{h}}$. in Altmanns Gemisch eingelegt worden waren, $\mathrm{n}$ ie primăr geschwărzte Körner in der Rinde fand, dass ich aber in Phosphornieren vom Menschen, die ähnlich behandelt wurden, das Fett primăr geschwärzt finden konnte: man darf darnach eine grosse Körnermenge in der Nebennierenrinde nicht als Produkt einer fettigen Degeneration der Rinde ausprechen.

Unter primärer Schwarrzung verstehe ich die unmittelbare Schwarzung von Fett durch Osmium ohne nachherige Alkoholeinwirkung.

Ueber die Fettverhăltnisse im Mark findet sich meines Wissens nur eine Angabe in der Literatur: die von Guarnieri und Magini (Arch. ital. de la biologie 1881: Etudes sur la fine structure des capsules surrenales). Diese beiden Autoren beschrieben folgendes: „Dans les préparations fixées avec les liquides osmiques on trouve constamment des corps cylindriques de substance fortement réfringente, entourrée par une mince couche d'autre substance noircie par l'acide osmique et de figure circulaire, semilunaire ou irregulière." Diese beiden Verfasser untersuchten die menschliche Nebenniere nebst solchen von Hund, Kaninchen und Rind. Diesen Befund kann ich nur bestătigen und folgendes hinzufügen.

In den Markzellen treten sichosmierende Körner bei menschlichen Embryonen erst bei $30-35 \mathrm{~cm}$ Körperlänge auf. Sie sind in diesem Stadium stets sehr klein, solid, höchst spärlich, nehmen bis zur Geburt und den ersten Lebensmonaten an 
Zahl kaum erheblich zu, wohl aber werden sie gröber. Sie sind noch um das fünfte Lebensjahr nicht in allen Zellen enthalten.

Um diese Zeit nun - im 5. Lebensjahr - treten in den Markzellen neben den soliden, schwarzen Körnern (in Paraffinschnitten) noch grössere Körner auf, die ein helles Centrum haben, welches von einem durch Osmium geschwarzten Ring umgeben ist. Nach der Pubertät, im 18.-19. Lebensjahre, finden wir in den Markzellen fast ausschliesslich die erwähnten Ringformen und nach dem 25. Lebensjahr ausschliesslich Ringformen: diese werden immer grösser (siehe Fig. 2.), der schwarze Ring wird immer schmäler, feinsaumartig. Mit fortschreitendem Alter treten an diesen Ringformen verschiedene Complicationen auf: man sieht einen kleineren Ring in einem weiteren liegen, oder ersterer kann ringsum von noch kleineren umgeben sein; der kleinere Ring kann eine excentrische Lage im weiteren annehmen, er kann sich ferner sehr stark erweitern, so dass er fast an den Saum des ausseren heranreicht. Je alter das untersuchte Individuum, desto complicierter und reichlicher werden diese Gebilde.

Man könnte bei diesen Formen an Auslangungsproducte denken; doch sind sie solches sicher nicht, da man Andeutungen jener Structuren schon im nativen Gefrierschnittprăparat sieht. Man findet genau dieselben Bilder auch in osmierten Gefrierschnitten, die gar nicht in irgendwelche fettlösende Flüssigkeit gekommen waren. Untersucht man aber Sudanpräparate auf diese Körner, so erscheinen letztere gleichmässig rot. Wie dieser Befund zu erklären ist, weiss ich nicht; das nächstliegende wäre allerdings, zwei verschiedene chemische Substanzen in den Ringgebilden anzunehmen, eine sich osmierende Mantelschicht und einen sich mit Sudan III färbenden Kern. Diese Erklärung ist mir jedoch nicht wahrscheinlich. In Paraffinschnitten konnte ich das Centrum dieser Ringgebilde in keiner Art gefarbt erhalten.

Diese beschriebenen Gebilde sind für die Markzellen $\mathrm{nach}$ dem 20. Lebensjahre typisch und man muss alle Zellen, die nach diesem Alter, insofern sie im Mark liegen und solide, mit Osmium sich schwärzende Körner führen, als versprengte Rindenzellen auffassen. Darnach würden auch die $\mathrm{Holm}$ 'schen Zellen von "zweifelhaft nervösem Charakter", wie es ja auch die meisten Autoren annehmen, als Rindenzellen aufzufassen sein. 
Auffallend gut färbten sich in allen embryonalen menschlichen Nebennieren Altmann's fuchsinophile Granula (R. Altmann: Die Elementarorganismen 1894.) (Siehe auch Virchow's Archiv, Bd. 135 : O. L u b a r s c h: Beiträge zur Histologie der von Nebennierenkeimen ausgehenden Nierengeschwülste, II, 179.) Bekanntlich lassen sich letztere nur in möglichst frisch eingelegten Organen gut conservieren, beziehungsweise färben. Vom intrauterinen Fruchttod abgesehen, bekam ich die Embryonen im Durchschnitt erst $24^{\mathrm{h}}$. nach dem Abortus oder der Frühgeburt zur Verfügung; trotzdem lionnte ich die fuchsinophilen Granula bei den meisten embryonalen Nebennieren gut darstellen. Nicht immer färbte sich alles, was im ungefärbten Präparat als Granulum erschien, mit Fuchsia rot; immerhin gelang es mir hăufig, den ganzen Granulainhalt zu färben.

Alle diese darstellbaren Granula sind in der Nuance und Intensität der Farbe gleich, dagegen ungleich in ihrer Grösse; im Durchschnitt erreichen sie die Grösse der osmierbaren Körner, ihre Form ist stets rund. Sie sind stets mehrschichtig und concentrisch um den Kern geordnet und zwar so, dass die kleinsten fuchsinophilen Granula dem Kerne zunächst liegen. In der Zellperipherie werden sie von der Schichte der osmierten Körner umgeben.

Liegen die Rindenzellen zu Haufen geordnet (Parenchymkörper); so finden wir, wie ich oben erwăhnte, an den zusammenstossenden Zellflächen keine oder nur vereinzelte Rindenkörner; im Fuchsinpräparat sieht man diese-Partien dicht mit fuchsinophilen Granula gefüllt. $\mathrm{Ob}$ sich die fuchsinophilen Granula in osmierbare Rindenkörner umwandeln oder nicht, dafür finde ich keine Anhaltspunkte: in Form und Grösse gleichen sich die fuchsinophilen und die osmierbaren Körner bis ins kleinste.

Legt man Paraffinschnitte von in Altmann fixierten $\mathrm{Ob}$ jecten auf einige Tage in Aether, so lösen sich schnell und ohne Rückstand sämtliche früher schwarz gewesenen Rindenkörner; daneben verschwindet auch eine grosse Zahl der fuchsinophilen Granula. Einzelne Granula dagegen erscheinen auch bei sehr langer Aetherwirkung auf Fuchsinfärbung wieder. Darnach dürften einzelne Granula gegen Aethereinwirkung resistenter sein und sich chemisch von den übrigen rascher sich lösenden unterscheiden. Dieser Befund würde für eine Präexistenz derselben in vivo sprechen. 
Stilling (Virchow's Archiv Bd. 109), Eberth (Stricker's Handbuch der Gewebelebre) und Grandry (Journal de l'anatomie et de la phys., 1867) beschreiben und bilden ab zum Teir Hohlräume (Stilling beim Rind, Grandry beim Menschen) zum Teil Spalten (Eb e $\mathrm{r} h$ ) in der ausseren Rindenzone der Nebenniere, die sie für prăexistent halten. Stilling hălt die erwăhnten Hohlrăume für Lymphgefässe, gestützt auf die Resultate bei Injectionen. Für die Präexistenz der Hohlräume führt er vor allem ihr stetes Vorkommen bei frischeingelegten Organen in verschiedene Fixierungsflüssigkeiten an. Gegenüber den $\mathrm{Ca}-$ pillaren betont er die Wandungslosigkeit der Hohlräume.

Aehnliche Hohlräume sah ich bei Embryonen von über $15 \mathrm{~cm}$ Lange in der aussersten Rindenperipherie; mit zunehmender Entwicklung des Fötus nehmen auch diese Hohlrăume an Zahl und Grösse zu und erreichen kurz vor der Geburt den Höhepunkt. Beim Neugeborenen sind sie wieder spärlich und sehr sparrlich sind sie in der zweiten Halfte des ersten Lebensjahres; beim Erwachsenen konnte ich sie nicht wieder finden.

Diese Hohlräume sind zumb Theil leer, zum Theil enthalten sie ein kernloses Netzwerk, das dem feinsten Reticulum der Parenchymkörper entsprechen könnte, z. Th. sind sie von Fåden durchzogen, die Fibrinfäden ăhneln, sich jedoch fărberisch nicht wie Fibrin verhalten; von Schleimfarbstoffen werden diese Fảden nicht gefärbt.

Diese Hohlräume sind bei allen Fixierungen und sowoht bei Paraffin als Celloidineinbettung vorhanden. Manchmal haben dieselben einen Belag von platten, cubischen Zellen, deren Plasma unmerklich heller als das der übrigen Rindenzellen ist, denen sie sonst in allem gleichen, und sicher nur als eigentümlich angeordnete Rindenzellen aufzufassen sind. Dieser einzellige Belag ist entweder continuierlich über den ganzen Hohlraum ausgebreitet oder er übergeht an einer Stelle unmerklich in die gew.öhnlichen Rindenzellen.

Bei anderen dieser Hohlraume, besonders, wenn sie Zelldetritus und Fäden enthalten, ist dieser Belag als etwas eigenes nicht vorhanden; der Hohlraum wird dann einfach von den Rindenzellen gebildet, die buchtig gegen das Lumen vorspringen und es scharf umgrenzen. Zwischen dem Hohlraum und daneben verlaufenden Capillaren liegt gewöhnlich nur die eine auskleidende Zellschichte. 
Diese Hohlräume sind beim Erwachsenen gar nicht, bei Kindern von einigen Monaten spärlich zu finden. Diese Beschränkung ihres Vorkommens spricht gegen die Meinung, es seien reine Macerations- oder Fixierungsartefacte; auch die eigentümliche Anordnung und Form der Auskleidungszellen in einzelnen derselben spricht dagegen. Man findet manchmal in ihnen auch rote Blutkörperchen, die ganz gut erhalten sind.

Wollte man die Abplattung jener Auskleidungszellen nur durch den Druck des sich ansammelnden oder verflüssigenden Inhalts erklaren, so ist zu bedenken, dass nur e in e Schichte der Zellen die Abplattung zeigt, dass die unmittelbar an ihr verlaufenden Capillaren strotzend mit Blut gefüllt sind und diese Auskleidungsschicht, wie erwahnt, öfters discontinuierlich ist. Auch sind diese Hohlräume seltener rein rund als öfters ausgebuchtet, mit einander communicierend.

Fortsetzungen der Hohlräume in die tieferen Rindenschichten oder in's Mark konnte ich weder an Quer- noch an Flachschnittserien verfolgen.

Bezüglich des Pigmen $\mathrm{ts}$ der Nebenniere ist folgendes zu bemerken: Man sieht dasselbe in der untersten Rindenzone, vor allem in der Reticularis bei gewöhnlichen Kern-Plasmafärbung ohne weiteres in Form gelblicher Körnchen. Mit $\mathrm{H}$ eid e $\mathrm{n}$ h a in 's Eisenbaematoxylin farbt sich das Nebennierenrindenpigment (und gewisse Markzellenbestandteile: Hultgreen und Andersson) tief schwarz in Form von runden Körnern. Ausserdem fand ich, dass sich dieses Rindenpigment mit Unna's polychromem Methylinblau und Glycerinätherdifferenzierung tief grasgrün färbt, wie auch die Markzellen diesen Ton annehmen. Auch geringe Pigmentmengen stechen bei dieser. Fürbung gegen die blauen Kerne und das ungefürbte Plasma ab. Diese Färbung gelingt jedoch nur an Objecten, die in nicht osmiumführenden Fixierungsflüssigkeiten erhărtet wurden.

Mit diesen Färbungen fand ich in embryonalen Nebennieren kein Pigment, wohl aber recht spärliches bei einem 6 Tage alten Kind.

Eine Abhängigkeit des Pigments vom Vorkommen der Rindenkörner konnte ich nicht nachweisen: das Pigment kommt sowohl in rindenkönerführenden als in rindenkörnerfreien Zellen vor 
Bei Fixierung der Objecte in Altmann's Gemisch verliert es die gelbe Farbe und wird schmutzig graugrünlich und ist von den tiefschwarzen Rindenkörnern leicht zu unterscheiden

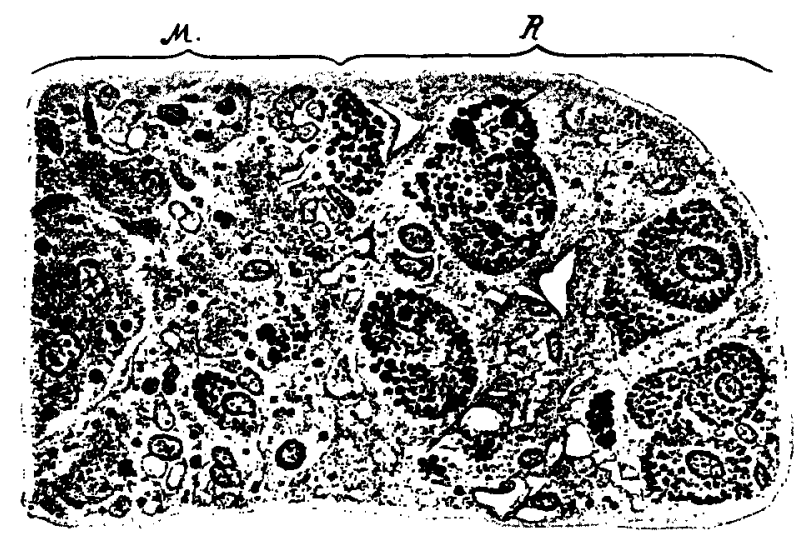

Figur 2.

(siehe Fig. 2):' Es scheinen jedoch die in Altmann's Gemisch fixierten Objecte weniger (im Durchschnitt) Pigment zu enthalten als entsprechende in Müller's Flüssigkeit erhärtete.

Extrahiert man die osmierten Schnitte mit Aether und färbt sie mit Heidenhain's Haematoxylin (siehe Fig. 3.), so erscheinen in der Reticularis an Stelle der (osmierten) schwarzen Körner grosse, helle Lücken, in dem wabigen Netz der Zellen aber die tiefschwarz gefärbten Pigmentkörner. Nach D o st o je wsk y (Archiv f. mikr. Anat., Bd. XXVII.: Ein Beitrag zur mikr. Anat. d. Neb. d. Säugetiere) entspricht dieses Netzwerk nicht der Filarmasse, sondern stellt das ganze Cytoplasma dar. Wegen dieser eigentümlichen Lage nun erscheint in Osmiumpräparaten weniger Pjgment als in Müllerpräparaten: es wird von den Rindenkörnern einfach verdeckt. Die Verbältnisse in den Pigment- und Rindenkörnerführenden Zellen sind also die, dass die Rindenkörner in den Plasma wabe $n$ liegen, in dem Plasma selbst aber liegen die Pigmentkörner.

Im Allgemeinen ist die Lage des Pigments $\mathrm{zu}$ den Rindenkörnern die, dass das Pigment in seiner Hauptmenge näher dem Kern, die Rindenkörner näher dem Zellrand zu liegen. (Siehe Fig. 2.) 
Nebennierenzellen frei in Gefassluminis liegend fand ich öfters, ebenso Rindenkörner. Es handelt sich dabei wohl immer um Kunstproducte.

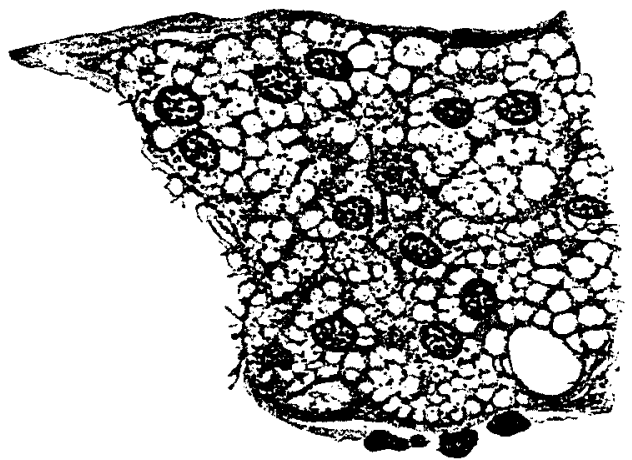

Figur 3.

Freibegrenzte, endothellose Zellhaufen aus dem Parenchym in die Gefässe ragend, wie sie Manasse (Virchow's Archiv, Bd. 135), über die Beziehungen d. Neben. zu d. Venen u. den venösen Kreislauf) beschreibt, konnte ich nicht finden.

Ich beschäftigte mich auch mit der Natur der Rindenkörner der menschlichen Nebenniere.

Eine Nachprüfung der Angaben von Rabl, Pfaundler. Alexander u. a. Autoren, die sich mit derselben Frage beschäftigten, machte ich nicht. Ich fand aber, entsprechend den Angaben der Autoren, dass sich die Rinden- und auch die Markkörner in Osmium primär nur bräunen, und sich erst nach Alkoholeinwirkung schwärzen. Die Markkörner stellen (falls sie schon Ringformen angenommen) auch in Gefrierschnitten Ringformen dar. (Einwirkung von Osmium 24 $4^{\mathrm{h}}$.)

Zum Vergleich untersuchte ich das pericapsulare Fett der Nebenniere, so wie das subepicardiale und das Degenerationsfett von Phosphornieren; dieselben schwärzen sich stets inOsmium auch oh ne Alkoholnachbehandlung. (Einwirkung v. Osmium 24.

Entwässert man einfach osmierte Nebennierengefrierschnitte (Formolhärtung) rasch in Alkohol und legt sie dann in Bergamotöl, Xylol, Aether, Petroläther, Chloroform, so löst Bergamotöl alle osmierten, d. h. braunen Körner sofort, Xylol in ungefahr ${ }^{1 / 4^{\mathrm{h}}}$, Aether rascher als Xylol, Chloroform und Petroläther nur zum Teil in $1 / 4^{\mathrm{h}}$. 
Das Fett (subepicardiales, pericapsuläres, Phosphorniere) löst sich (bei analog behandelten Objecten) in Bergamotöl rapid, in den anderen angeführten Lösungsmitteln dagegen in $1 / 4^{\mathrm{h}}$. fast gar nicht.

Behandelt man die nur osmierten Gefrierschnitte mit Alkohol durch $24^{\text {h. }}$, so werden, wie erwähnt, die braunen Rindenkörner schwarz, ebenso die braunen Markringe; das pericapsulăre u. a. Fett bleibt schwarz, wie es gewesen.

Extrahiert man $n$ u n die Schnitte mit den erwähnten fettlösenden Mitteln, so findet man, dass Bergamotöl nach wie vor rapid alle s osmierte löst, die anderen Mittel aber (in $1 / 4^{\mathrm{h}}$.) viel weniger von dem geschwărzten Fett lösen, als sie von dem nur gebrăunten lösten; das Petroläther und das Chloroform erscheinen ganz wirkungslos, Aether löst die Nebennierenkörner bedentend rascher als Xylol. Das pericapsulare u. a. Fett bleibt in der angegebenen Zeit, ausser in Bergamotöl, fast intact.

L ewins ohn (Archiv f. mikrosk. Technik, Bd. XVIII.) gab eine Fettfärbungsmethode in Celloidinschnitten an, die sich in ibrem Wesen an die Haematoxylin-Markscheidenfärbung anlehnt. Sie besteht in langdauerndem Belassen der Organe in Müller's Flüssigkeit, Einbettung derselben in Celloidin durch aufsteigenden Alkohol ohne vorheriges Auswaschen, Schneiden, Uebertragen der Schnitte direkt aus Alkohol in die EssigsaureHaematoxylinlösung, Nachbehandeln mit Kalium permauganicum, Differenzierung in Oxalsäure.

Lewinsohn fand nach dieser Methode das "Fett" in den interstitiellen Hodenzellen blau gefärbt. Ueber die Resultate von Färbungsversuchen anderer Fette berichtet er nicht, gibt aber seine Methode als allgemeingültig an.

Diese Methode versuchte auch ich und fand folgendes:

Gefrierschnitte durch lange gemüllerte Nebennieren lassen ihren Körnergehalt nach der beschriebenen Methode färben. Auch nach Einbettung lange gemüllerter Nebennieren in Celloidin und oben erwähnter Behandlung bekam ich die „Fett"-Körner der Nebenniere gefärbt.

Gefrierschnitte durch lange gemüllertes subepicardiales Fett und Phosphornierenfett ergaben mit obiger Methode k eine Fettfarbung, ebensowenig entsprechende Celloidinschnitte.

Ich legte nun Celloidinschnitte von lange gemüllerten 
Nebennieren, subepicardialem Fett und Phosphornieren in Altmann's Gemisch auf $48^{\mathrm{h}}$. bei $38^{\circ} \mathrm{C}$. und fand nach abspülen derselben mit Wasser das Nebennieren- "Fett“ tief schwarz gefärbt, nicht aber das subepicardiale Fett und die Phosphorniere.

Darnach zu schliessen, besteht also $z$ wischen dem Nebennierenfett und anderen Fetten tatsa chlich ein Unterschied.

Zum Scblusse der Arbeit sei es mir gestattet, dem Vorstande des Insitutes, Herrn Hofr. Prof. W eichselba um den aufrichtigsten Dank für die Anregung der vorliegenden Arbeit. und für die gütige Zuweisung des Materials zu danken.

\section{Erklärung zu den Abbildungen.}

Fig. 1. Schnitt durch die Nebennierenrinde eines $30 \mathrm{~cm}$ langen menschlichen Fötus. Fixierung in Altmann's Gemisch. Vesuvinnachfärbung. a, $a^{1}$ die im Text erwähnten hellen Zellen, die eine davon kernlos.

Fig. 2. Rinden-Markgrenze eines 47-j. Mannes. $R$.=Rinde mit den Osmiumkörner- (schwarz) und Pigment- (grau) führenden Zellen der Zona reticularis. $M r=M a r k$; in seinen Zellen die eigentümlichen Ringformen der Markkörner.

Fig. 3. Aus der Zona reticularis eines in Altmann's Gemisch fixierten Paraffinschnittes von dem vorigen Falle, dessen Rindenkörner mit Aether extrahicrt wurden. Nachfärbung des Pigmentes mit. Heidenhain's Eisenhaematoxylin. 